\title{
Optical and infrared spectroscopic analysis of the post-AGB star candidate IRAS 19386+0155 ${ }^{\star}$
}

\author{
C. B. Pereira ${ }^{1}$, S. Lorenz-Martins ${ }^{2}$, and M. Machado ${ }^{1}$ \\ 1 Observatório Nacional, Rua José Cristino, 77, CEP 20921-400, São Cristóvão, Rio de Janeiro, Brazil \\ e-mail: claudio@on.br \\ 2 GEMAC/Departamento de Astronomia/Observatório do Valongo/UFRJ, Ladeira Pedro Antônio 43, \\ CEP 20080-090, Rio de Janeiro, Brazil
}

Received 29 October 2003 / Accepted 24 March 2004

\begin{abstract}
This work reports high-resolution spectroscopic stellar parameters and abundance analysis of the post-AGB star IRAS 19386+0155. The analysis of the high resolution spectrum shows that it has an effective temperature of $T_{\text {eff }}=6800 \pm$ $100 \mathrm{~K}$ and a surface gravity of $\log g=1.4 \pm 0.2$. The abundance analysis reveals IRAS $19386+0155$ to be a metal-poor object having $[\mathrm{Fe} / \mathrm{H}]=-1.1$. Carbon, oxygen and nitrogen are found to be slight underabundant than solar, and so are the $\alpha$-elements $(\mathrm{Mg}, \mathrm{Si}$ and $\mathrm{Ca})$. The analysis of ISO spectra revealed an oxygen-rich envelope and showed crystalline silicate features. The dust envelope of this object was modelled using a Monte Carlo type numerical method and amorphous and crystalline silicate grains were considered. The photospheric temperature obtained through the optical spectroscopy was used to constrain these models. The Spectral Energy Distribuition (SED) of this object is broad and extensive suggesting the presence of a dust disk around IRAS $19386+0155$.
\end{abstract}

Key words. stars: AGB and post - AGB - stars: abundances

\section{Introduction}

The photospheric chemical composition of post-AGB stars which has been widely investigated in recent years reveals a rather complex picture (see van Winckel 2003, for the most recent review). Post-AGB stars can be basically divided into four categories, (i) hot post-AGB stars in which some are carbon deficient (McCausland et al. 1992) while others are not such as LSIV $-12^{\circ} 111$ and HD 341617 (Ryans et al. 2003); (ii) those that present signatures of s-process enrichment in their optical spectra, these could also be called " $21 \mu \mathrm{m}$ stars" because of the presence of a C-rich circumstellar feature at $21 \mu \mathrm{m}$; (iii) those that exhibit strong iron depletion such as HR 4049 which has $[\mathrm{Fe} / \mathrm{H}]=-4.9$ (Waelkens et al. 1991) these are also known as "metal-poor binary post-AGB stars"; and (iv) those that are O-rich with a double peaked SED and are not enriched in sprocess elements such as HR 4912 (Luck et al. 1990). During the last phase of the evolution of a low mass star on AGB, the surface composition changes when material is brought to the surface (third dredge-up) from the He-burning shell; there is an increase in the amounts of carbon and elements synthesized

^ Based on observations made with the $1.52 \mathrm{~m}$ telescope at the European Southern Observatory (La Silla, Chile) under the agreement with the CNPq-Observatório Nacional (Brazil) Based on observations with ISO, an ESA project with instruments funded by ESA Member States (especially the PI countries: France, Germany, the Netherlands and the UK) and with the participation of ISAS and NASA. by slow neutron capture reactions. Stars that are in the second category above mentioned have their surface compositions changed thanks to these reactions.

However there are some post-AGB stars that show neither enrichment of elements created by slow neutron capture reactions nor carbon enrichment. Among them are the O-rich stars of which quite a few have already been investigated in the literature and to which the present paper is dedicated. We concentrated our efforts on investigating IRAS $19386+0155$, based on spectroscopy in the optical and in the infrared. We carried out high-resolution optical spectroscopy to derive the stellar parameters and photospheric abundances. In the IR we used ISO SWS (Infrared Satellite Observatory - Short Wavelength Spectrograph) data to describe the main spectroscopic features and to model its circumstellar envelope. We will use the spectroscopic data in both wavelength ranges for our discussion of the evoluitonary status of IRAS $19386+0155$.

\section{Observations}

The high-resolution spectrum of IRAS 19386+0155 analysed in this work was obtained at the $1.52 \mathrm{~m}$ ESO telescope on October 10, 2000 with the FEROS (Fiberfed Extended Range Optical Spectrograph) echelle spectrograph (Kaufer et al. 1999). The FEROS spectral resolving power is $R=48000$ corresponding to 2.2 pixels of $15 \mu \mathrm{m}$ and the wavelength coverage is from $4000 \AA$ to $9200 \AA$. The nominal S/N ratio was 


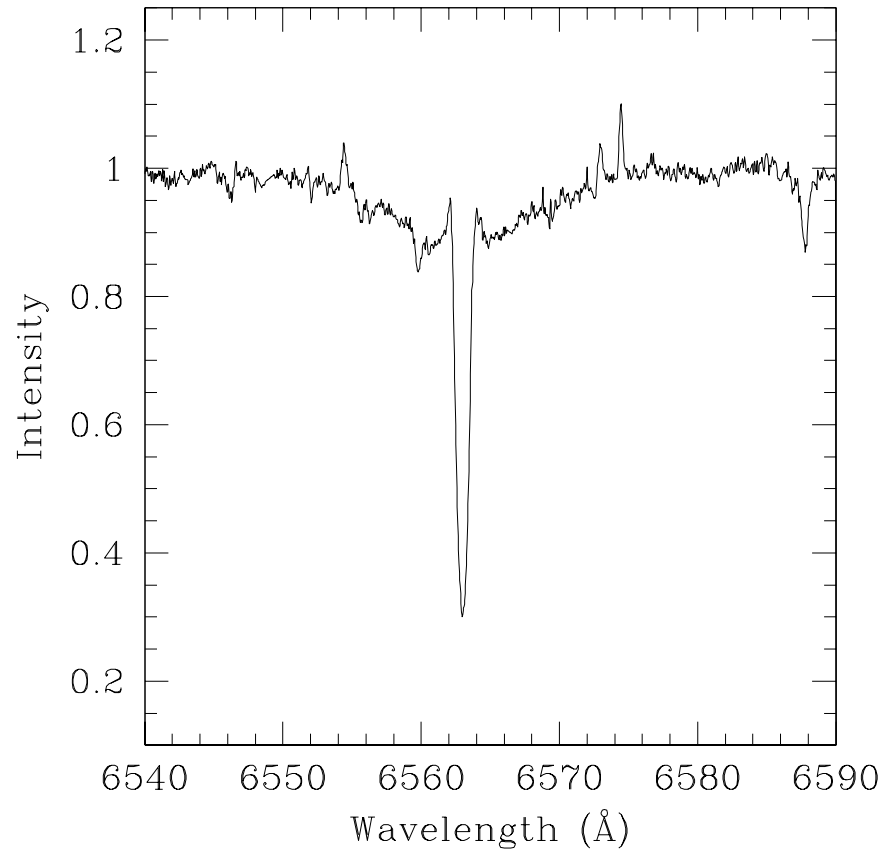

Fig. 1. Normalized spectrum of IRAS $19386+0155$ around $\mathrm{H} \alpha$.

evaluated by the measurement of the rms flux fluctuation in selected continuum windows, and a typical value achieved was $\mathrm{S} / \mathrm{N} 100-150$, after $2 \times 3600 \mathrm{~s}$ of integration time. Figure 1 shows the spectral region near $\mathrm{H} \alpha$.

\section{Analysis and results}

\subsection{Line selection, measurement and oscillator strengths}

The absorption spectrum of IRAS $19386+0155$ shows many atomic absorption lines of Fe I and Fe II as well as transitions due to CI, NI, SI, CaI, Si I, MgI, NaI, ZnI, Y II and BaII. We have chosen a set of lines that are sufficiently unblended to yield reliable abundances. The selected lines are listed in Table 1 for the Fe I and Fe II, and in Table 2 for the other elements. In Table 1 we list the Fe I and Fe II lines employed in the analysis and also the lower excitation potential, $\chi(\mathrm{eV})$, of the transitions, the $g f$-values and the measured equivalent widths. The latter were obtained by fitting gaussian profiles to the observed lines. The $g f$-values for the Fe I and Fe II lines in Table 1 were taken from Lambert et al. (1996).

\subsection{Physical properties of the star: Effective temperature and gravity}

The determination of stellar atmospheric parameters, effective temperature $\left(T_{\text {eff }}\right)$, surface gravity $(\log g)$, microturbulence $(\xi)$ and $[\mathrm{Fe} / \mathrm{H}]$ (throughout, we use the notation $[\mathrm{X} / \mathrm{H}]=$ $\log (N(\mathrm{X}) / N(\mathrm{H}))_{\star}-\log \left(N(\mathrm{X}) / N(\mathrm{H})_{\odot}\right)$ is a prerequisite for the determination of the photospheric abundance. The gravity was determined by forcing Fe I and Fe II to yield the same iron abundance at the selected effective temperature. The microturbulent velocity was determined by forcing the abundance determined from individual Fe I lines to show no dependence
Table 1. Observed Fe I and Fe II absorption lines in IRAS $19386+0155$.

\begin{tabular}{|c|c|c|c|c|}
\hline Element & $\lambda(\AA)$ & $\chi(\mathrm{eV})$ & $\log g f$ & $W_{\lambda}(\mathrm{m} \AA)$ \\
\hline \multirow[t]{49}{*}{$\mathrm{Fe} \mathrm{I} \ldots$} & 4903.31 & 2.88 & -0.93 & 95 \\
\hline & 4946.39 & 3.37 & -1.01 & 39 \\
\hline & 4966.09 & 3.33 & -0.84 & 75 \\
\hline & 5022.24 & 3.98 & -0.49 & 48 \\
\hline & 5083.34 & 0.96 & -2.91 & 72 \\
\hline & 5090.77 & 4.26 & -0.36 & 41 \\
\hline & 5110.41 & 0.00 & -3.76 & 94 \\
\hline & 5121.64 & 4.28 & -0.72 & 36 \\
\hline & 5125.12 & 4.22 & -0.08 & 86 \\
\hline & 5133.69 & 4.18 & 0.20 & 117 \\
\hline & 5150.84 & 0.99 & -3.00 & 68 \\
\hline & 5162.27 & 4.18 & 0.08 & 114 \\
\hline & 5198.71 & 2.22 & -2.14 & 43 \\
\hline & 5202.34 & 2.22 & -1.84 & 84 \\
\hline & 5216.27 & 1.61 & -2.12 & 91 \\
\hline & 5242.49 & 3.63 & -0.97 & 24 \\
\hline & 5281.79 & 3.04 & -0.83 & 90 \\
\hline & 5307.36 & 1.61 & -2.97 & 53 \\
\hline & 5339.93 & 3.27 & -0.68 & 100 \\
\hline & 5341.02 & 1.61 & -1.95 & 142 \\
\hline & 5353.37 & 4.10 & -0.68 & 47 \\
\hline & 5364.87 & 4.45 & 0.23 & 94 \\
\hline & 5367.47 & 4.42 & 0.44 & 117 \\
\hline & 5369.96 & 4.37 & 0.54 & 120 \\
\hline & 5383.37 & 4.31 & 0.65 & 133 \\
\hline & 5400.50 & 4.37 & -0.10 & 49 \\
\hline & 5410.91 & 4.47 & 0.40 & 107 \\
\hline & 5434.52 & 1.01 & -2.12 & 158 \\
\hline & 5445.04 & 4.39 & 0.04 & 77 \\
\hline & 5506.78 & 0.99 & -2.80 & 109 \\
\hline & 5554.90 & 4.55 & -0.38 & 42 \\
\hline & 5563.60 & 4.19 & -0.84 & 32 \\
\hline & 5569.62 & 3.42 & -0.49 & 96 \\
\hline & 5576.09 & 3.43 & -0.85 & 76 \\
\hline & 5633.95 & 4.99 & -0.12 & 18 \\
\hline & 5686.53 & 4.55 & -0.45 & 27 \\
\hline & 5762.99 & 4.21 & -0.41 & 56 \\
\hline & 6024.06 & 4.55 & -0.06 & 62 \\
\hline & 6065.48 & 2.61 & -1.53 & 51 \\
\hline & 6136.61 & 2.45 & -1.40 & 101 \\
\hline & 6137.69 & 2.59 & -1.40 & 77 \\
\hline & 6393.60 & 2.43 & -1.43 & 45 \\
\hline & 6411.65 & 3.65 & -0.66 & 69 \\
\hline & 6419.95 & 4.73 & -0.09 & 26 \\
\hline & 6421.35 & 2.28 & -2.01 & 48 \\
\hline & 6430.85 & 2.18 & -2.01 & 18 \\
\hline & 6592.91 & 2.72 & -1.47 & 52 \\
\hline & 6593.87 & 2.44 & -2.42 & 21 \\
\hline & 6677.99 & 2.69 & -1.22 & 42 \\
\hline \multirow[t]{8}{*}{ Fe II ... } & 5132.66 & 2.81 & -4.00 & 87 \\
\hline & 5325.56 & 3.22 & -3.17 & 173 \\
\hline & 5414.05 & 3.22 & -3.62 & 97 \\
\hline & 5425.25 & 3.20 & -3.21 & 125 \\
\hline & 6084.10 & 3.20 & -3.80 & 56 \\
\hline & 6149.25 & 3.89 & -2.72 & 116 \\
\hline & 6416.92 & 3.89 & -2.68 & 105 \\
\hline & 6432.68 & 2.89 & -3.58 & 107 \\
\hline
\end{tabular}


Table 2. Other absorption lines studied and their respective abundance.

\begin{tabular}{|c|c|c|c|c|c|c|c|}
\hline$\lambda(\AA)$ & Species & $\chi(\mathrm{eV})$ & $\log g f$ & Ref. & $W_{\lambda}(\mathrm{m} \AA)$ & $\log \epsilon$ & {$[\mathrm{X} / \mathrm{H}]$} \\
\hline 7111.480 & $\mathrm{C}_{\mathrm{I}}$ & 8.64 & -1.32 & LRB & 32 & 7.71 & \\
\hline 7113.180 & CI & 8.64 & -0.95 & LRB & 58 & 7.65 & \\
\hline 7115.190 & C I & 8.64 & -0.89 & LRB & 60 & 7.64 & \\
\hline 7116.990 & C I & 8.64 & -1.08 & LRB & 53 & 7.74 & \\
\hline \multirow[t]{3}{*}{7119.660} & $\mathrm{CI}_{\mathrm{I}}$ & 8.64 & -1.31 & LRB & 49 & 7.93 & \\
\hline & & & & & & 7.74 & -0.78 \\
\hline & & & & & & \pm 0.12 & \\
\hline 8736.040 & $\operatorname{Mg} I$ & 5.94 & -0.34 & WSM & 110 & 7.14 & \\
\hline 5772.150 & Si I & 5.08 & -1.81 & LB85 & 41 & 7.65 & \\
\hline 6145.080 & Si I & 5.62 & -1.48 & LB85 & 25 & 7.53 & \\
\hline 7405.790 & Si I & 5.61 & -0.82 & LB85 & 74 & 7.43 & \\
\hline 8556.800 & Si I & 5.87 & -0.73 & LB85 & 110 & 7.55 & \\
\hline \multirow[t]{3}{*}{8648.430} & Si I & 6.21 & -0.30 & LB85 & 136 & 7.52 & \\
\hline & & & & & & 7.53 & -0.01 \\
\hline & & & & & & \pm 0.20 & \\
\hline 5588.760 & $\mathrm{Ca} \mathrm{I}$ & 2.53 & -0.05 & LB85 & 163 & 5.95 & \\
\hline 5594.470 & $\mathrm{Ca} \mathrm{I}$ & 2.52 & -0.31 & LB85 & 107 & 5.92 & \\
\hline 5601.280 & $\mathrm{Ca} \mathrm{I}$ & 2.53 & -0.63 & LB85 & 34 & 5.64 & \\
\hline 5857.450 & $\mathrm{Ca} \mathrm{I}$ & 2.93 & 0.07 & LB85 & 112 & 5.91 & \\
\hline 6122.220 & $\mathrm{Ca} \mathrm{I}$ & 1.89 & -0.20 & LB85 & 159 & 5.54 & \\
\hline 6439.070 & $\mathrm{Ca} \mathrm{I}$ & 2.53 & -0.05 & LB85 & 160 & 5.94 & \\
\hline \multirow[t]{3}{*}{6471.660} & Ca I & 2.51 & -0.67 & LB85 & 45 & 5.81 & \\
\hline & & & & & & 5.81 & -0.55 \\
\hline & & & & & & \pm 0.16 & \\
\hline 4848.230 & Cr II & 3.86 & -1.15 & MFW & 181 & 4.64 & \\
\hline 5237.330 & Cr II & 4.07 & -1.16 & MFW & 181 & 4.81 & \\
\hline \multirow[t]{3}{*}{5313.560} & Cr II & 4.07 & -1.56 & MFW & 107 & 4.85 & \\
\hline & & & & & & 4.77 & -0.90 \\
\hline & & & & & & \pm 0.11 & \\
\hline 4810.530 & $\mathrm{Zn} \mathrm{I}$ & 4.06 & -0.39 & BG80 & 61 & 4.09 & \\
\hline \multirow[t]{2}{*}{4810.530} & $\mathrm{Zn} \mathrm{I}$ & 4.06 & -0.17 & BG80 & 78 & 4.04 & \\
\hline & & & & & & 4.07 & -0.53 \\
\hline 4883.680 & Y II & 1.08 & 0.07 & LB85 & 154 & 1.20 & \\
\hline 5087.420 & Y II & 1.08 & -0.17 & LB85 & 100 & 1.16 & \\
\hline 5200.410 & Y II & 0.99 & -0.57 & H82 & 36 & 0.95 & \\
\hline \multirow[t]{3}{*}{5402.780} & Y II & 1.84 & -0.38 & LB85 & 21 & 1.22 & \\
\hline & & & & & & 1.13 & -1.11 \\
\hline & & & & & & \pm 0.13 & \\
\hline
\end{tabular}

References to Table 2:

BG80: Biémont \& Godefroid (1980); H82: Hannaford et al. (1982).

LB85: Luck \& Bond (1985).

LRB: Lambert et al. (1982).

MFW: Martin et al. (1988).

WSM: Wiese et al. (1969).

on equivalent width. The excitation equilibrium was solved for by requiring that the slope of the correlation between the $\mathrm{Fe} I$ abundances and the excitation potential of the measured lines should be zero. The solution thus found is unique, depending only of a set of Fe I, II lines and the atmospheric model employed, and yields as a by-product the metallicity of the star $[\mathrm{Fe} / \mathrm{H}]$. The atmospheric parameters were determined based on LTE model atmospheres of Kurucz (1993) 

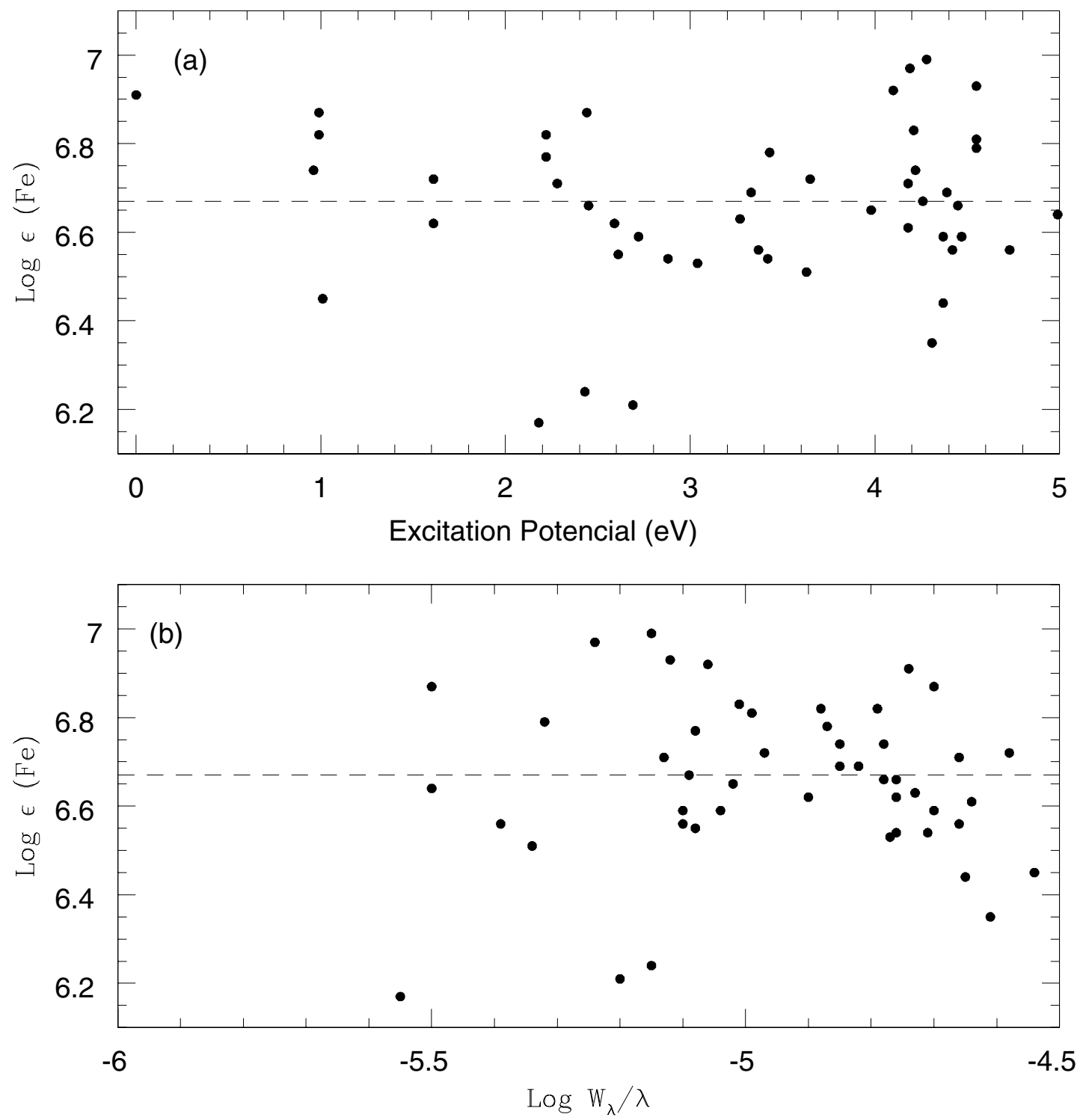

Fig. 2. The abundance of Fe by number $\epsilon(\mathrm{Fe})$ vs. excitation potential a) and $\log \left(W_{\lambda} / \lambda\right)$ b) for the 57 lines of Fe I,II employed in the determination of $T_{\text {eff }}, \xi$ and $\epsilon(\mathrm{Fe})$ in IRAS $19386+0155$. The calculation was done adopting the values $T_{\text {eff }}=6800 \mathrm{~K}, \log g=1.4, \epsilon(\mathrm{Fe})=6.67$ and $\xi=$ $8.4 \mathrm{~km} \mathrm{~s}^{-1}$. The absence of any trend of the individual abundances with excitation potential and microturbulence shows that a correct value for $T_{\text {eff }}$ and microturbulence velocity was chosen.

using the spectral analysis code MOOG (Sneden 1973). The final adopted atmospheric parameters were $T_{\text {eff }}=(6800 \pm$ $100 \mathrm{~K}), \log g=(1.4 \pm 0.2) \mathrm{cm} / \mathrm{s}^{2}, V_{\mathrm{t}}=(8.4 \pm 0.2) \mathrm{km} \mathrm{s}^{-1}$ and $[\mathrm{Fe} / \mathrm{H}]=-(1.08 \pm 0.14)$. Figure 2 shows the diagrams corresponding, respectively, to the abundances of the individual Fe I lines plotted against the lower excitation potential and the reduced line strength following the best parameters above mentioned. The internal errors in our adopted effective temperatures $\left(T_{\text {eff }}\right)$ and microturbulent velocity $(\xi)$ can be determined from the uncertainty in the slope of the Fe I abundance versus excitation potential and Fe I versus reduced equivalent width $\left(W_{\lambda} / \lambda\right)$ relation. The standard deviation in $\log \mathrm{g}$ was set by changing this parameter around the adopted solution until the difference between Fe I and Fe II mean abundance differed by exactly one standard deviation of the $[\mathrm{Fe} / \mathrm{H}]$ mean value.

\subsection{Abundance analysis}

Abundances of chemical elements of IRAS 19386+0155 were determined for an LTE model atmosphere. The line-synthesis code MOOG was used to carry out the calculations. The results are given in Table 2 which lists the lines employed for each species, the abundances derived from each line, and the ratio $[\mathrm{X} / \mathrm{H}]$. The abundances of nitrogen, sulfur and oxygen were obtained by spectrum synthesis. The nitrogen and sulfur abundances were obtained using lines between $8680 \AA$ and $8695 \AA$ while for oxygen the triplet at $6156 \AA$ was used. The analysis of these lines gives final abundances for nitrogen, sulfur and oxygen of $7.28,6.76$ and 8.43 respectively. The $g f$-values for nitrogen and sulfur were taken from Bond \& Luck (1987) and for oxygen from Venn (1993). Figures 3 and 4 show the synthesized spectra for these elements. 


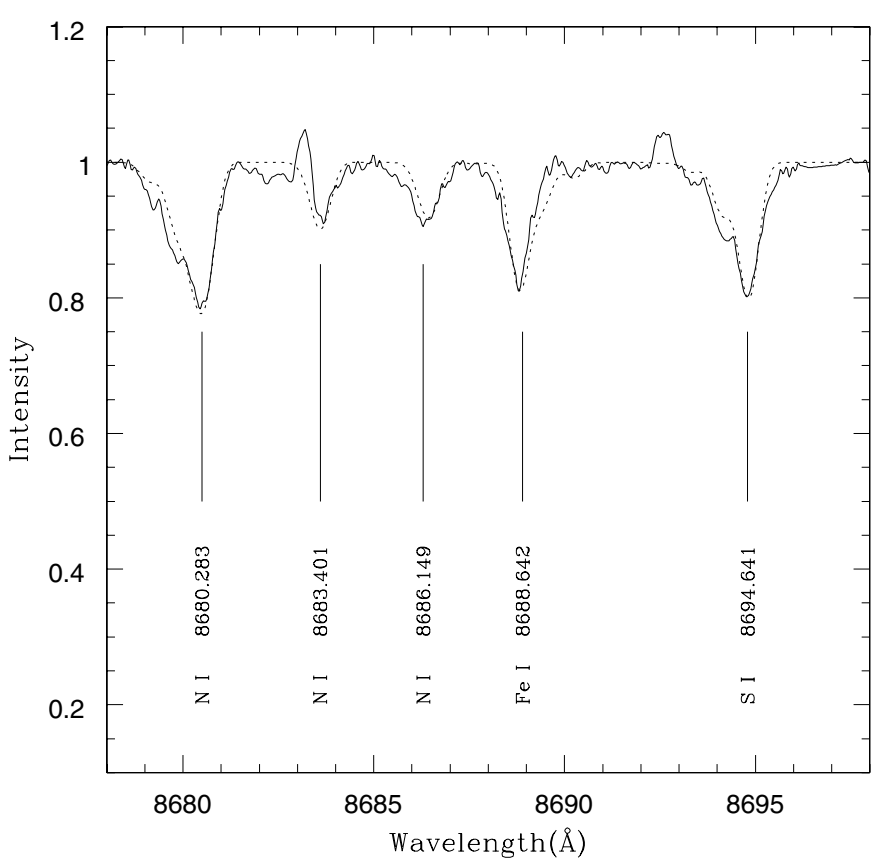

Fig. 3. Fit of the synthesized spectrum to the $8680 \AA-8695 \AA$ region of IRAS $19386+0155$. The thick line represents the observed spectrum and the dotted line the synthesized spectrum calculated for $\log \epsilon(\mathrm{N})=$ $7.28([\mathrm{~N} / \mathrm{Fe}]=+0.21), \log \epsilon(\mathrm{S})=6.76([\mathrm{~S} / \mathrm{Fe}]=+0.28), T_{\text {eff }}=$ $6800 \mathrm{~K}$ and $\log g=1.4$.

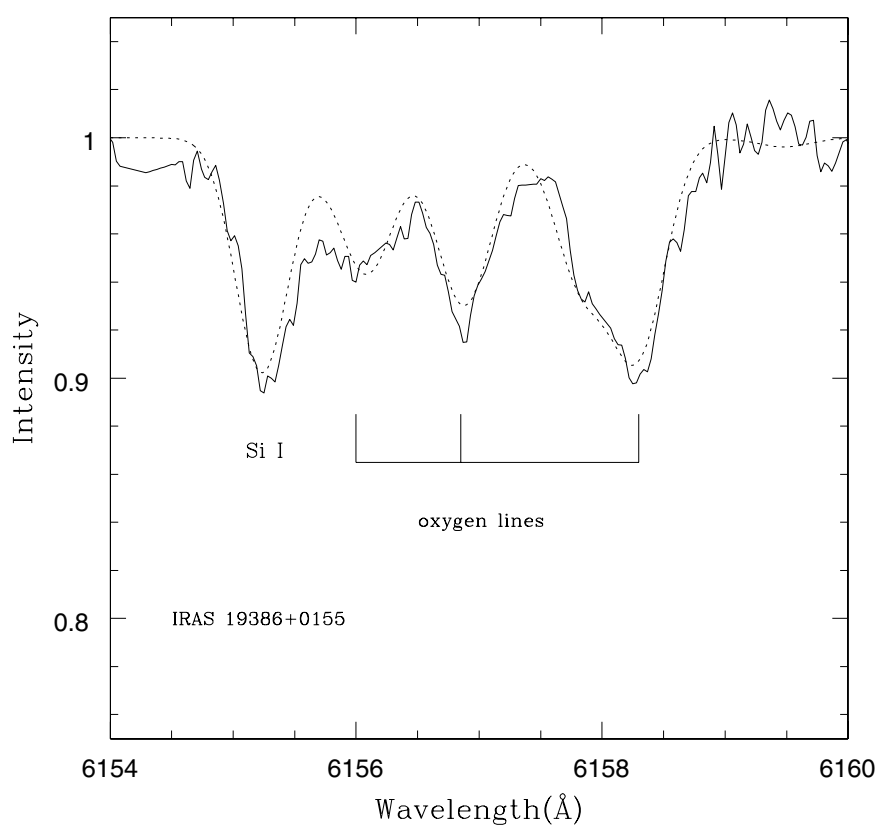

Fig. 4. Fit of the synthesized spectrum to the $6152 \AA-6158 \AA$ region of IRAS $19386+0155$. The thick line represents the observed spectrum and the dotted line the synthetic spectrum calculated for $\log \epsilon(\mathrm{O})=$ $8.63([\mathrm{O} / \mathrm{Fe}]=+0.65), T_{\text {eff }}=6800 \mathrm{~K}$ and $\log g=1.4$.

The internal scatter in the derived abundances of each element that has than two lines is also given in Table 2. The uncertainties in the derived abundances for the program star are dominated by three main sources: the $g f$-values, the equivalent width measurements and the stellar parameters. The uncertainties in the abundances due to errors in the stellar atmospheric

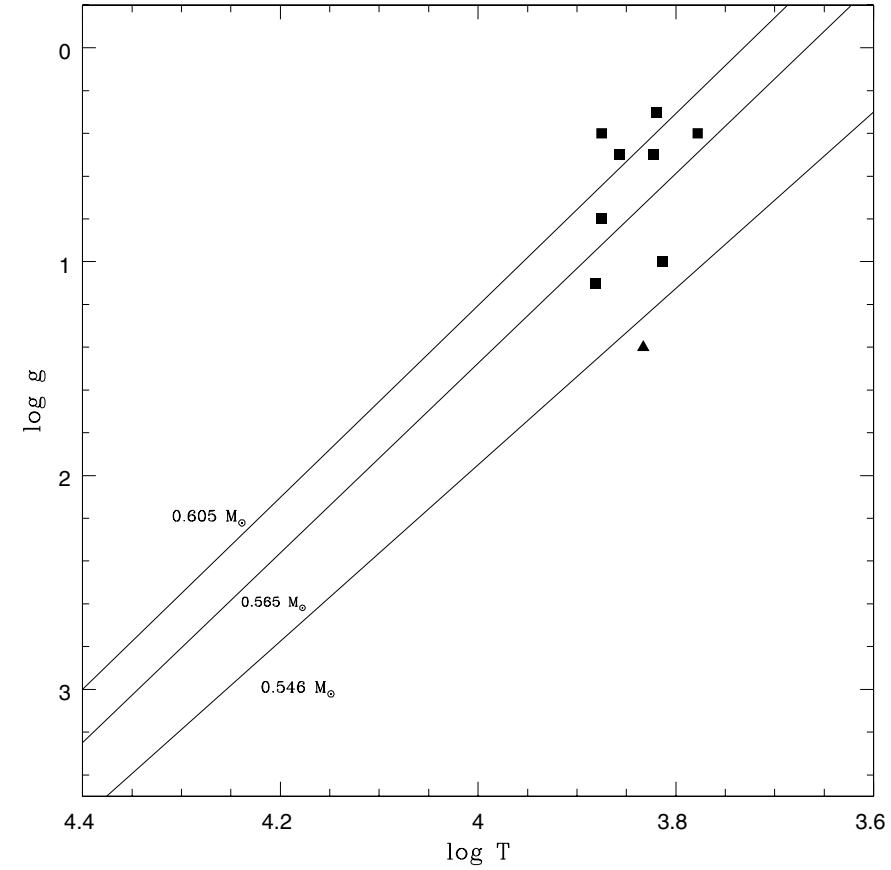

Fig. 5. Logarithmic surface gravity versus effective temperature diagram showing the location of IRAS $19386+0155$ (filled triangle) and of previously identified post-AGB objects (filled squares). Data for post-AGB stars were taken from Table 1 of Luck (1993). Post-HB tracks of Schönberner (1983) and Blöcker \& Schönberner (1990) are also shown.

parameters were computed by changing these parameters by their standard errors and calculating the changes in the element abundances. The uncertainties due to the atmospheric parameters, $T_{\text {eff }}: \pm 110 \mathrm{~K} ; \log g: \pm 0.2$ dex and $V_{\mathrm{t}}: 0.4$ in the derived abundances are: carbon, 0.2 ; nitrogen, 0.1 ; silicon, 0.1 ; calcium, 0.1 and oxygen 0.2 .

\section{Discussion}

\subsection{Stellar parameters and evolutionary tracks}

With the derived stellar parameters of $T_{\mathrm{eff}}=6800$ and $\log g=1.4$, the post-AGB star IRAS $19386+0155$ can be plotted in the $\log g-\log T$ plane and compared to posthorizontal branch evolutionary tracks of Schönberner (1983) and Blöcker \& Schönberner (1990). These are indicated in Fig. 5 as solid lines for $0.546 M_{\odot}, 0.565 M_{\odot}$ and $0.605 M_{\odot}$. The position of other post-AGB stars previously studied is also given in Fig. 5. The parameters derived here suggest that the mass of IRAS $19386+0155$ is $M \sim 0.55 M_{\odot}$.

\subsection{The abundance pattern}

Below we discuss the abundance pattern, comparing it with previous studies for post-AGB stars. In Fig. 6 we show the CNO abundances for several classes of objects such as Population I supergiants (Luck \& Bond 1989; Luck \& Lambert 1985), planetary nebula (PNe) (Kingsburgh \& Barlow 1994) and other post-AGB stars. The thick line represents the ratio $\mathrm{C} / \mathrm{O}=1.0$. In this figure we use different symbols for 


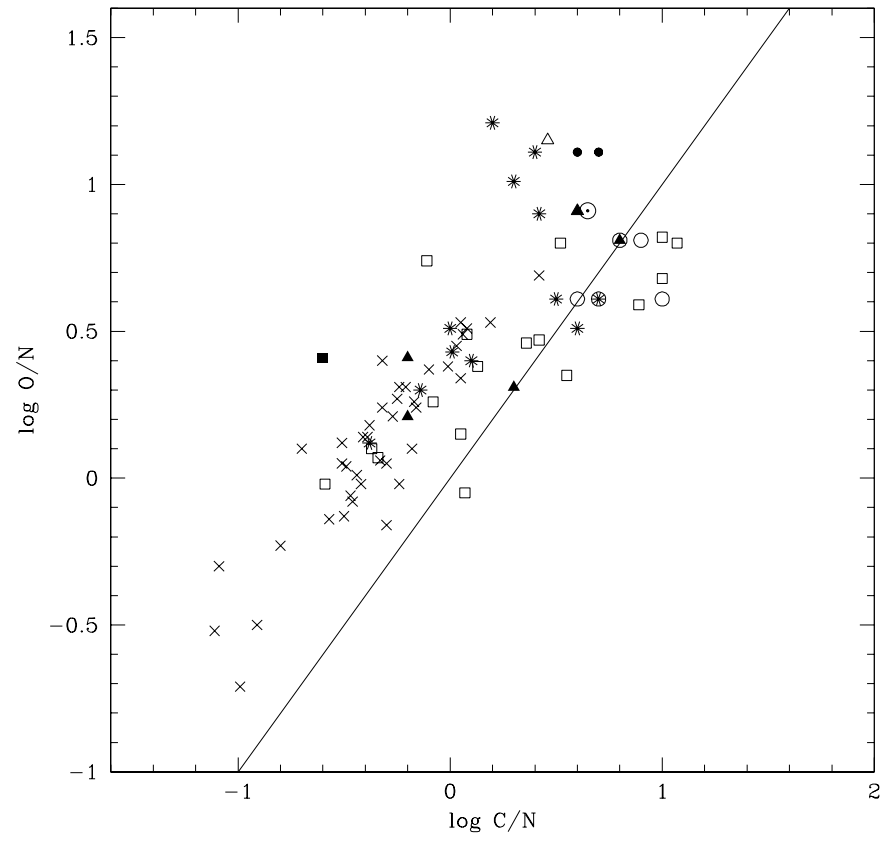

Fig. 6. Relative abundance $\mathrm{O} / \mathrm{N}$ against $\mathrm{C} / \mathrm{N}$ for post $\mathrm{AGB}$ stars, planetary-nebula and supergiants. Open squares, PNe; diagonal cross points, Pop. I supergiants; open circles, " $21 \mu \mathrm{m}$ stars"; filled triangles, metal-poor binary post-AGB stars; asterisks, Group B post-AGB stars and HD 172481; filled square point, ROA 24; hot post-AGB stars, filled circles and open triangle, IRAS 19386+0155. Data on PNe are from Kingsburgh \& Barlow (1994); on Pop. I supergiants, from Luck \& Bond (1989) and Luck \& Lambert (1985); on post-AGB stars, from Luck et al. (1990), van Winckel (1997), van Winckel \& Reyniers (2000), Reyniers \& van Winckel (2001) and Trams et al. (1993); on ROA 24, Gonzalez \& Wallerstein (1992); on hot post-AGB stars, Ryans et al. (2003) and on IRAS 19386+0155, this work.

different types of post-AGB stas depending on their abundance pattern, those that display the circumstellar feature at $21 \mu \mathrm{m}$ (van Winckel \& Reyniers 2000); metal-poor binary post-AGB stars (Trams et al. 1993) and post-AGB stars that are not s-process enriched (Luck et al. 1990; van Winckel 1997). Figure 6 also shows the position of IRAS $19386+0155$ in the $\mathrm{O} / \mathrm{N}-\mathrm{C} / \mathrm{N}$ plane. Luck (1993) divided post-AGB stars in two groups, A and B. Those that belong to Group A all have [element/Fe] much higher than unity, reflecting that the ratio $[\mathrm{Fe} / \mathrm{H}]$ is not primordial but is a result of grain condensation (Trams et al. 1993), and are members of binary systems. These stars are also known as "metal-poor binary post-AGB stars". Those that belong to Group B, are post-AGB stars that do not show extreme iron deficiencies and do not show a large overabundance of carbon and elements created by slow neutron capture reactions.

Figure 6 clearly shows that Pop. I supergiants show traces of nitrogen enrichment due to $\mathrm{CNO}$-cycling. PNe are found on either sides of the $\mathrm{C} / \mathrm{O}=1$ line. Those that are on the same side of the $\mathrm{C} / \mathrm{O}=1.0$ line as the Pop. I supergiants seem to dispaly only $\mathrm{CNO}$-processed while those on the other side of the line show carbon enhancement of carbon due to He burning. On the same side of the the "21 $\mu \mathrm{m}$ stars" also have $\mathrm{C} / \mathrm{O}>1.0$ The CNO abundances

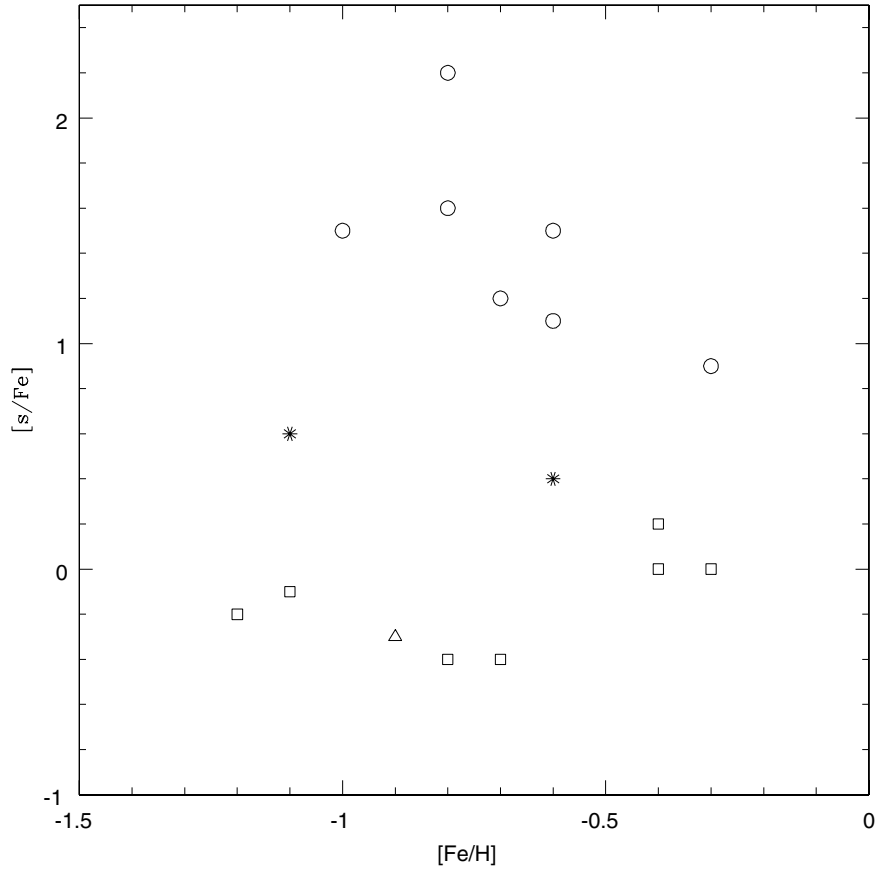

Fig. 7. The mean of s-process elements given by the $[\mathrm{s} / \mathrm{Fe}]$ ratio as a function of metallicity for several post-AGB stars. Open circles, "21 $\mu \mathrm{m}$ stars"; open squares, non-enriched post-AGB stars; asterisks, HD 172481 and HR 7671 and open triangle, IRAS 19386+0155.

in these metal-poor binary post-AGB stars are basically solar, while some display slight nitrogen enrichment. Figure 6 also shows the position of two hot post-AGB stars analysed by Ryans et al. (2003). IRAS $19386+0155$ is close to the three other post-AGB stars: SAO 239853, HD 133656 and HR 4912. Although these three stars have modest carbon enrichment with $[\mathrm{C} / \mathrm{Fe}]=+0.4,+0.3$ and +1.0 respectively for HR 4912, SAO 239853 and HD 133656 they do not show significant s-process overabundances, $[\mathrm{s} / \mathrm{Fe}]=-0.4$, -0.4 and -0.2 respectively for SAO 239853, HD 133656 and HR 4912. For IRAS $19386+0155$ we found $[\mathrm{s} / \mathrm{Fe}]=-0.3$ and $[\mathrm{C} / \mathrm{Fe}]=+0.1$.

Figure 7 shows the mean heavy-element abundance as given by $[\mathrm{s} / \mathrm{Fe}]$ vs. $[\mathrm{Fe} / \mathrm{H}]$. In this figure all the " $21 \mu \mathrm{m}$ stars" have $[\mathrm{s} / \mathrm{Fe}] \geq 1.0$ and they follow the trend already seen in both classes of objects that are intrinsically and extrinsically s-process enriched (van Winckel \& Reyniers 2000; Pereira \& Junqueira 2003), e.g., an increasing [s/Fe] with decreasing metallicity. Figure 7 shows that there is a clear distinction between the heavily enriched " $21 \mu \mathrm{m}$ stars" and nonenriched objects. Figure 7 also shows that the abundance seen in IRAS $19386+0155$ again is similar to that of the post-AGB stars SAO 239853 and HD 133656. Note the positions of HD 172481, which was analysed by Reyniers \& van Winckel (2001) and HR 7671 (Luck et al. 1990). They both have moderate s-process overabundances, but underabundances of carbon $([\mathrm{C} / \mathrm{Fe}]=-0.01$ and -0.3 , respectively for HD 172481 and HR 7671).

Figure 8 gives the same information as Fig. 7 but for the $\alpha$-elements $(\mathrm{Mg}, \mathrm{Si}$ and $\mathrm{Ca})$ viz. $[\alpha / \mathrm{Fe}]$ vs. $[\mathrm{Fe} / \mathrm{H}]$ for the same stars as in Fig. 7. We do not see any trend in 


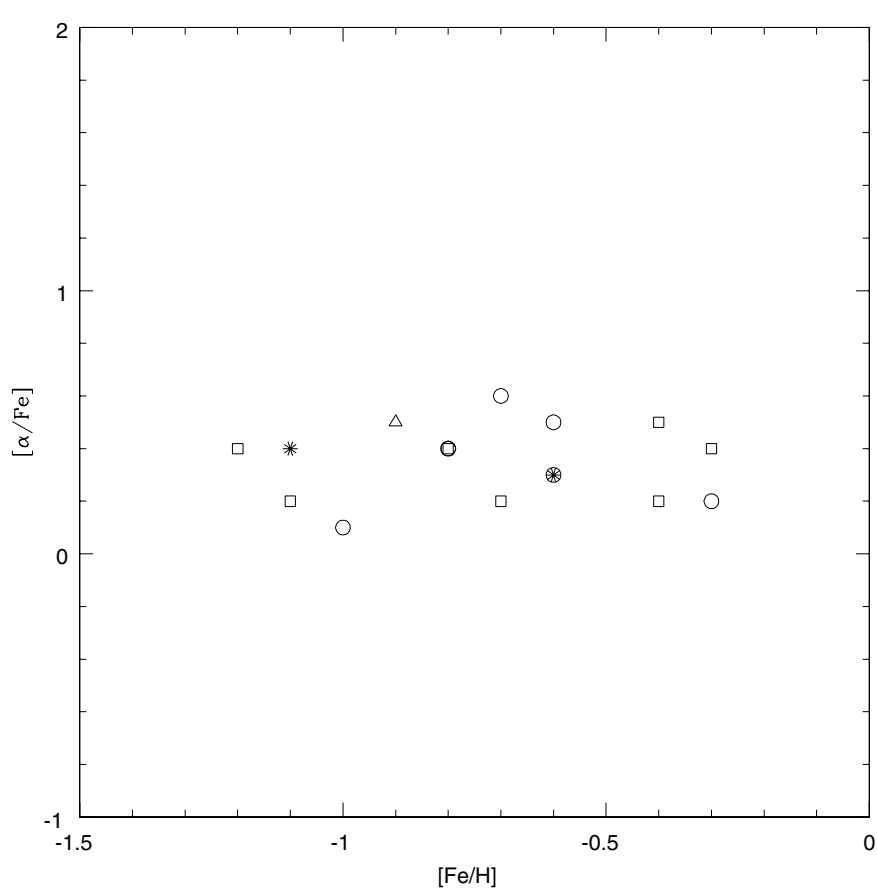

Fig. 8. The mean of $\alpha$-elements given by the $[\alpha / \mathrm{Fe}]$ ratio as a function of metallicity for several post-AGB stars. Symbols have the same meaning as in Fig. 7.

this ratio with metallicity or difference between the different groups of post-AGB stars. The $\alpha$-overabundances seen in IRAS $19386+0155$ as well as in other post-AGB stars follow the trend seen in disk and halo stars of the same metallicity (Gratton \& Sneden 1987).

From the abundance analysis we may conclude that IRAS $19386+0155$ displays an abundance pattern which is very similar to that of three post-AGB stars, SAO 239853, HD 133656 and HR 4912. Their positions in the $\log \mathrm{O} / \mathrm{N}-$ $\log \mathrm{C} / \mathrm{N}$ plane are above the $\mathrm{C} / \mathrm{O}=1$ line, in a region whre $\log \mathrm{O} / \mathrm{N} \geq 1.0$. Among these three stars, HR 4912 has the lowest metallicity, $[\mathrm{Fe} / \mathrm{H}]=-1.2$ (Figs. 7 and 8 ). In order to compare also the infrared region of these stars with IRAS 19386+0155, we search for ISO SWS01 spectra. Unfortunately, only SAO 239853 has been observed and presents a very noisy spectra which prevents us from reaching a conclusion about the presence or absence of crystalline silicate features (as seen in IRAS 19386+0155 spectra, Sect. 5). However, the shape of the ISO SWS01 spectrum is similar to that of IRAS 19386+0155; both peak around $23 \mu \mathrm{m}$. As will be shown in Sect. 5.2, the SED of IRAS 19386+0155 does not shows the double peak emission seen in some post-AGB stars, such as IRAS 19114+0002 (=HD 179821, Fig. 9b), an oxygen-rich post-AGB star. Unfortunately there are no available oxygen and nitrogen abundances for this star (Thévenin et al. 2000) so that we could not compare it with IRAS $19386+0155$. Neither star, IRAS $19386+0155$ or HD 179821, is s-process enriched. Finally, note the position of two hot post-AGB stars analysed by Ryans et al. (2003), LSIV $-12^{\circ} 111$ and HD 341617 . These two stars have similar $\log \mathrm{C} / \mathrm{N}$ and $\log \mathrm{O} / \mathrm{N}$ ratios as IRAS $19386+0155$. In fact, they maybe hotter analogues of the F-type post-AGB stars (Ryans et al. 2003).

\section{The Infrared region and SED of IRAS 19386+0155}

\subsection{ISO SWSO1 spectrum}

In addition to the photosphere analysis, in the next subsections we will describe the infrared spectrum of IRAS $19386+0155$ comparing it with other post-AGB stars using LRS and especially ISO SWS01 spectra, and we will also model its circumstellar envelope. We aim to determine the chemistry and also some properties of the circumstellar dust shell. The IRAS LRS spectrum of this source is noisy however, the silicate feature at $9.7 \mu \mathrm{m}$ seems to be self-absorbed and the $20 \mu \mathrm{m}$ is absent. The spectral resolution of ISO SWS AOT01 spectra is relatively high for dust spectroscopy $(\lambda / \Delta \lambda \approx 1500)$. The spectra $(\lambda \lambda 2.36-45.3 \mu \mathrm{m})$ consist of 12 sub-spectra with sightly overlapping wavelength coverage, each observed by 12 detectors. Each spectrum is scanned twice, the so called up- and downscan (de Graauw et al. 1996). In this work we use the highly processed spectrum of IRAS $19386+0155$ provided by Sloan et al. (2003). This spectrum is characterized by the absence of the silicate feature at $9.7 \mu \mathrm{m}$ and the presence of a few emission lines, probably due to crystalline silicate dust grains. Overall this spectrum presents a steep rise from 14 to $20 \mu \mathrm{m}$ and peaks at approximately at $23 \mu \mathrm{m}$.

We have compared the ISO SWS spectrum of IRAS 19386 with spectra of two other post-AGB sources: HD 44179 and IRAS 10215-5916 (HD 302821). The first is a metalpoor binary post-AGB star and the second is an oxygen-rich post-AGB star. The ISO SWS spectrum of IRAS 19386+0155 shows no sign of PAH or of the $21 \mu \mathrm{m}$ features. Figure $9 \mathrm{a}$ shows a comparison between the ISO SWS spectra of HD 44179 (1) and IRAS 19386+0155 (2). In this figure, the wavelengths of strong PAH features are indicated by with long-dashed vertical lines. In HD 44179 they are present at 3.3, 6.2, 7.7, 8.6 and $11.2 \mu \mathrm{m}$ and at $\approx 30 \mu \mathrm{m}$ there is also an emission bump probably due to crystalline silicates, also visible in the IRAS 19386 spectrum. The PAH lines are absent in the IRAS 19386 spectrum but the shape of the SED is very similar, although IRAS 19386 shows an emission bump towards $20 \mu \mathrm{m}$, probably due to amorphous silicate grains. Figure $9 \mathrm{~b}$ again gives the spectrum of IRAS $19386+0155$, this time compared to that of IRAS 10215-5916(1). The SED of the latter source is clearly double-peaked, and can be divided into two parts. For wavelengths shorter than $5 \mu \mathrm{m}$ the spectrum is dominated by the photosphere radiation, and above $5 \mu \mathrm{m}$ by the thermal radiation of the dust. In contrast to some oxygen- and carbon-rich post-AGB stars which present a double-peak energy distribution IRAS $19386+0155$ presents a broad SED suggesting the presence of hot dust grains $\left(T_{\mathrm{d}} \approx 700-800 \mathrm{~K}\right)$ which are responsible for the excess continuum at about $5 \mu \mathrm{m}$. On the other hand the excess at about $20 \mu \mathrm{m}$ can be due to cold dust grains $\left(T_{\mathrm{d}} \approx 200 \mathrm{~K}\right)$. We will discuss this later.

Now we will describe some features present in the ISO SWS spectrum. Figure 10a shows the $2-4 \mu \mathrm{m}$ region. On 

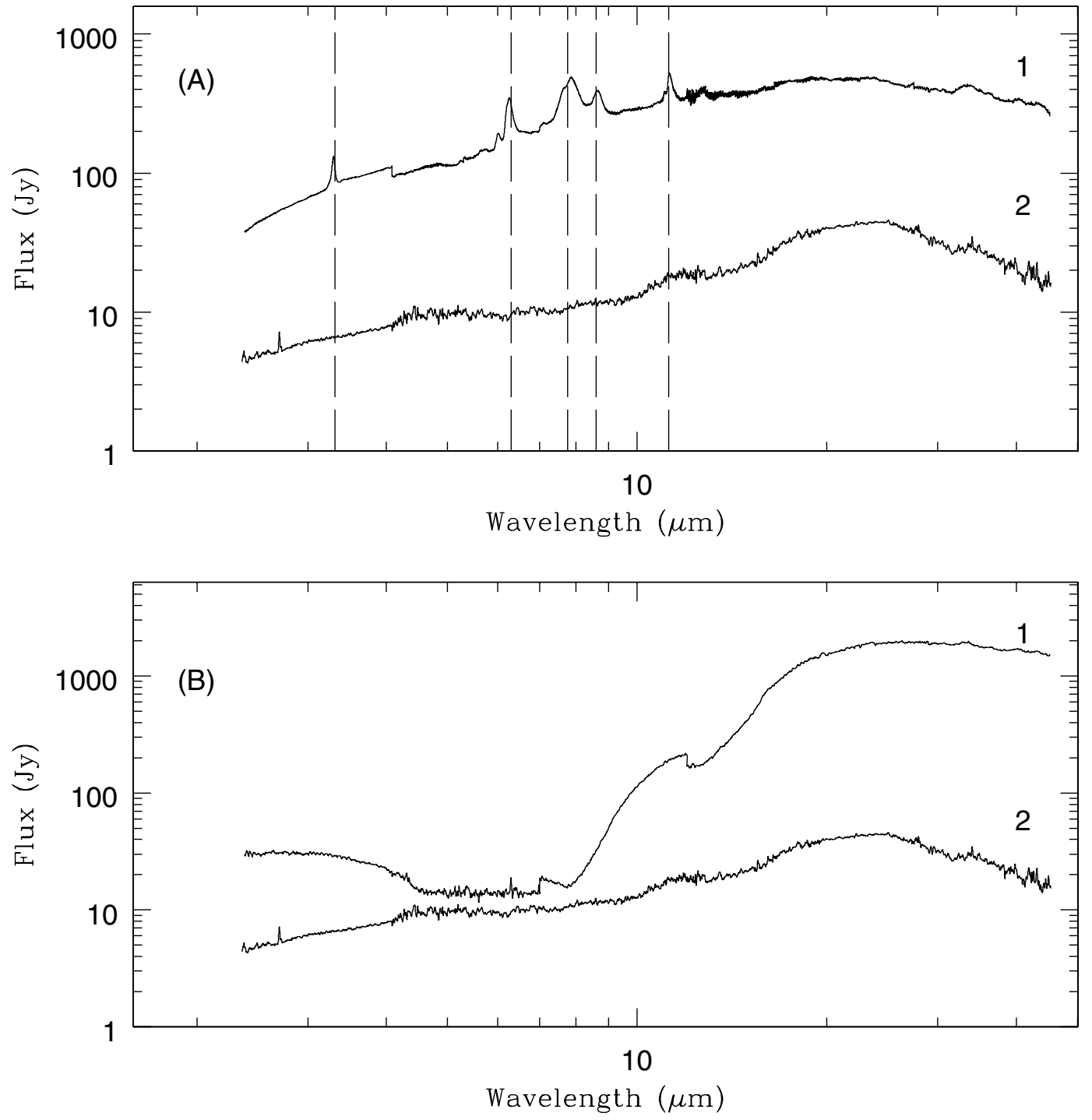

Fig. 9. ISO SWS spectra of IRAS 19386+0155 (2 in both figures) and two other post-AGB stars. a) Shows the spectrum of HD 44179 (1); b) that of IRAS 10215-5916 (HD 302821) (1).

the short-wavelength side of this spectrum there is one unidentified emission feature at $2.6 \mu \mathrm{m}$. The absence of $\mathrm{H}_{2} \mathrm{O}$ ice $\mathrm{ab}-$ sorption features at 3.1 and $6.0 \mu \mathrm{m}$ is stressed in this figure. The region between $4-7 \mu \mathrm{m}$ is probably dominated by water lines (see Cami 2002). We show some of them in Fig. 10b as well as the absorption at $4.25 \mu \mathrm{m}$, which is attributed to $\mathrm{CO}_{2} . \mathrm{CO}$ and $\mathrm{SiO}$, both fundamental absorption bands, may be present in this region, but only the lines of lower excitation.

The region 7 to $20 \mu \mathrm{m}$ is presented in Fig. 11a. As we said before, there is no sign of the amorphous silicate feature at $9.7 \mu \mathrm{m}$ in this region. A broad emission feature is present at $18 \mu \mathrm{m}$. This feature was first observed by Cami (2002) in a sample of oxygen-rich AGB stars. He suggested that this emission could be due to some crystalline grain. More recently, Molster et al. (2002) suggested that forsterite grains are also present at this wavelength. A similar feature is also observed in the ISO SWS01 spectrum of the post-AGB IRAS 19306+1407.

Figure $11 \mathrm{~b}$ shows the region between $\lambda \lambda 20-46 \mu \mathrm{m}$. In this region we can see some faint emission features which can be due to crystalline silicate grains. Vertical dashed lines indicate the crystalline silicate features as described in Molster et al. (2002). Forsterite is indicated by O (Olivine), enstatite by $\mathrm{P}$ (Pyroxene) and Diopside by $\mathrm{D}$. The identifications of crystalline silicate features is based on the laboratory measurements of Koike et al (2000) and Jäger et al. (1998). At $43 \mu \mathrm{m}$ we can suggest the presence of crystalline $\mathrm{H}_{2} \mathrm{O}$-ice; however, it should be noted that we cannot confirm this identification without having found a second emission at $62 \mu \mathrm{m}$. Unfortunately, there is no observation with the ISO Long Wavelength Spectra (LWS) for IRAS $19386+0155$. In addition to the emission features there is an absorption bump between $\lambda \lambda 26-34 \mu \mathrm{m}$ often attributed to a blend of crystalline enstatite and forsterite grains. A similar feature is present also in the ISO SWS spectrum of the post-AGB IRAS $20572+4919$. Two other supposed absorption features are seen at $31.8 \mu \mathrm{m}$ and $33.4 \mu \mathrm{m}$. Molster et al. (2002) have identified crystalline forsterite as carrier of the $33.6 \mu \mathrm{m}$ feature. 

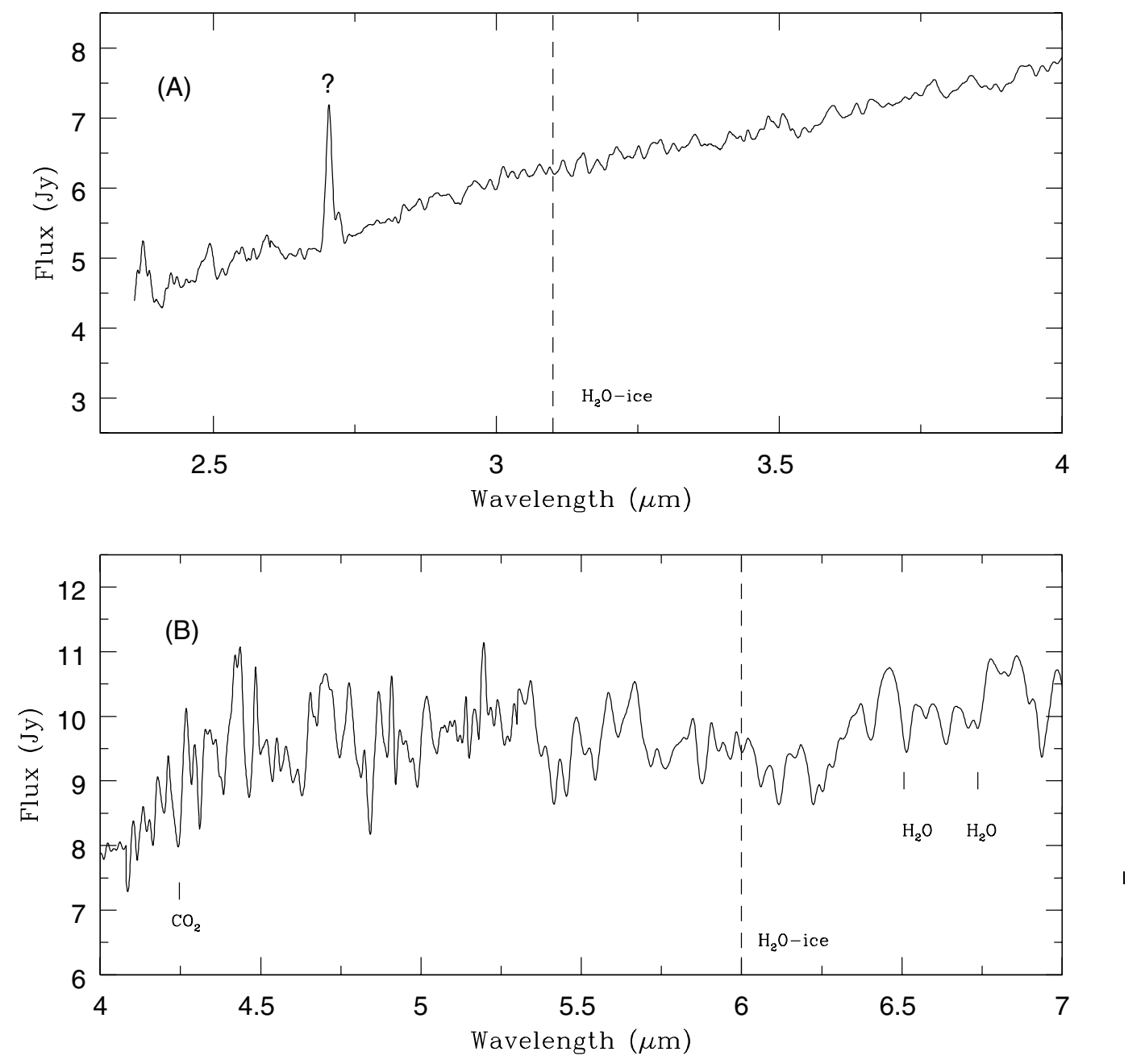

Fig. 10. a) ISO SWS $2.5-4 \mu \mathrm{m}$ region for IRAS $19386+0155$ showing the unidentified emission feature; b) ISO SWS $4.5-8.5 \mu \mathrm{m}$ region showing the absence of the $\mathrm{H}_{2} \mathrm{O}$-ice feature.

\subsection{Modelling}

In this section we present a method of numerical simulation to solve the problem of radiative transfer in spherical circumstellar dust envelopes and its application to IRAS $19386+0155$. We aim to calculate dust envelope parameters for IRAS $19386+0155$ and to verify if the temperature obtained through the spectroscopic analysis can be used to describe the SED. We would also like to show that the SED of IRAS $19386+0155$ cannot be described with a spherically symmetric geometry. The numerical treatment of radiative transfer in a spherical envelope was described in Lorenz-Martins $\&$ Pompeia (2000). A Monte Carlo scheme is used to represent the propagation of radiative energy photon by photon. For each interaction between a "photon" and a grain, a fraction of the energy is stored (absorption) and the remaining part is scattered according to the scattering diagram. Two species of grains are used simultaneously and in all processes they interact with each other. The stellar radiation leads to a first distribution of dust temperature and the thermal radiation from grains is simulated, giving after several iterations the equilibrium temperature. Here we assume that the numerical density of both species of grains decreases as $r^{-2}$, which corresponds to an expansion at constant velocity. The grains can be present at same or at different distances of the central star.

The following physical quantities are required for a first guess and are subsequently fixed by fitting the infrared/near infrared flux:

- the effective temperature of the central star, $T_{\text {eff }}$ (the star is supposed to radiate as a black-body);

- the grain sizes ( $a$ ) and refractive indices for all wavelengths;

- the extinction opacity, $\tau_{\text {ext }}$ at $\lambda=1 \mu \mathrm{m}$;

- the inner radii of the dust envelope $\left(R_{\text {Sil }}\right.$, inner radius for the amorphous silicate grains; $R_{\text {Crys }}$, inner radius for crystalline silicate grains).

The models give us not only the spectral repartition of the total flux but also of its different components (direct, scattered, thermal for each species of grain), as well as the angular distribution of the radiation fields and the radial dependence of dust temperature. The absorption and scattering efficiencies, as well as the albedo for the grains were calculated using the Mie theory and the optical constants tabulated in the literature. We used the optical constants calculated by David \& Pégourié (1995) to describe amorphous silicate grains, and those of Jäger et al. (1998) to describe crystalline grains. In addition to the 

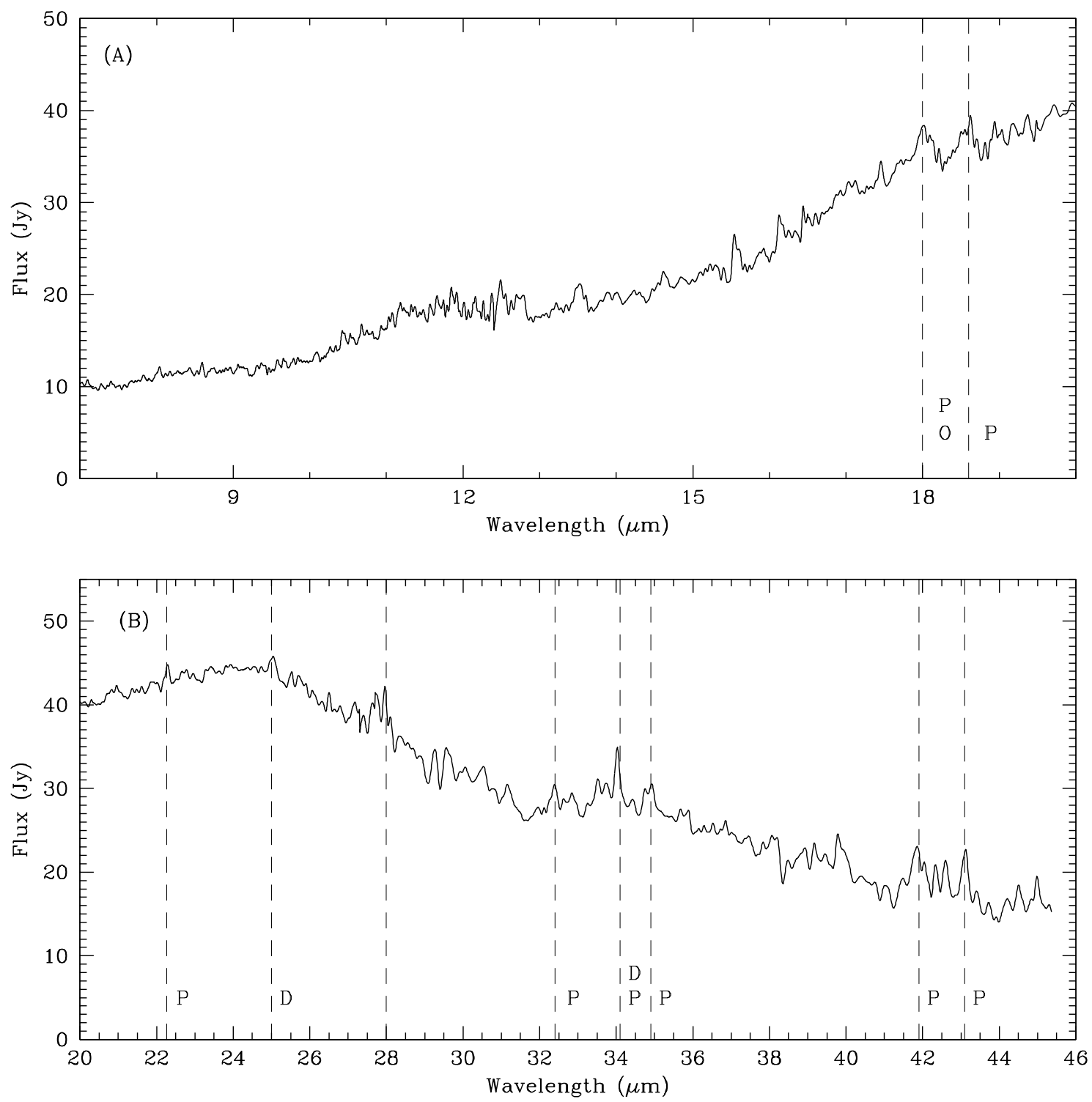

Fig. 11. a) ISO SWS 7-20 $\mu \mathrm{m}$ region: some emission features are present at about $18 \mu \mathrm{m}$. b) ISO SWS $20-45 \mu \mathrm{m}$ region: some further emission features are indicated. A broad absorption bump at $26-34 \mu \mathrm{m}$ can be seen. See text.

models with two species of grains, we have also calculated models using only amorphous silicate grains.

About a hundred models were calculated to fit the SED of IRAS 19386+0155. The results for seven of these are presented in Table 3. The name of each model is followed by the effective temperature (in K) of the central stars; Cols. 3 and 4 present the inner radii of the dust envelope where each species of grain is present $\left(R_{\mathrm{Sil}}\right.$ and $\left.R_{\mathrm{Crys}}\right)$ and Col. 5 the outer radii (in units of stellar radii $\left(R_{*}\right)$ ). The code also provides the choice of different distances of the central stars for each compound. The size grains (in $\AA$ ) are presented in Cols. 6 and 7, and the abundance ratio Sil/Crys is presented in Col. 8. The temperature of the hottest grains is present in Cols. 9 and 10, and Col. 11 presents the extinction opacity. The last part of Table 3 presents an amorphous silicate model. In this model we considered only amorphous grains with two different sizes. The LRS IRAS spectrum was taken from http://www . iras. ucalgary. ca/ volk/getlrs. plot.html and IRAS fluxes as well as optical and near infrared photometry were taken from Fouqué et al. (1992) and Arkhipova et al. (2000). All these data were used to constrain the models. Figures 12 and 13 show some of the fits.

In Fig. 12a three models are presented. The effective temperature of the central star $\left(T_{\mathrm{eff}}\right)$, the optical depth $\left(\tau_{9 \mu \mathrm{m}}\right)$, the outer radius $\left(R_{\mathrm{o}}\right)$ of the dust envelope, the size of the grains $\left(a_{\text {Sil }}, a_{\text {Crys }}\right)$ and the Sil/Crys ratio were fixed, see Table 3. We varied only the inner radii $\left(R_{\text {Sil }}, R_{\text {Crys }}\right)$ and all models present a detached envelope. Dotted line represents model A in which $R_{\mathrm{Sil}}=250 R_{*}$ and $R_{\mathrm{Crys}}=320 R_{*}$. The temperature obtained for silicate grains varies between $97 \mathrm{~K}$ to $205 \mathrm{~K}$, and for crystalline grains varies between $88 \mathrm{~K}$ to $236 \mathrm{~K}$. In this model $90 \%$ of the total energy is due to diffuse energy in the wavelength range of $\lambda=0.8 \mu \mathrm{m}$ to $\lambda=3.4 \mu \mathrm{m}$. At $\lambda=0.3 \mu \mathrm{m}$ and $\lambda=0.3 \mu \mathrm{m}$ the stellar radiation is responsible for practically all the energy. The contribution of thermal radiation by dust grains begins at $\lambda=5 \mu \mathrm{m}$, however the diffuse energy is 
Table 3. Results of Modelling. The effective temperature $\left(T_{\mathrm{eff}}\right)$ of central stars is given in $\mathrm{K}$ as are the temperatures of dust grains $\left(T_{\mathrm{Sil}}\right.$ and $\left.T_{\mathrm{Crys}}\right)$; inner radii of hottest grains $\left(R_{\mathrm{Sil}}\right.$ and $\left.R_{\text {Crys }}\right)$ and outer radius $\left(R_{\mathrm{o}}\right)$ of the envelope are given in $R_{*}$; grain sizes are given in $\AA$; and $\tau_{\mathrm{ext}}$ is taken at $\lambda=9.0 \mu \mathrm{m}$. For more details see text.

\begin{tabular}{lcccccccccc}
\hline \hline Model & $T_{\text {eff }}$ & $R_{\text {Sil }}$ & $R_{\text {Crys }}$ & $R_{\mathrm{o}}$ & $a_{\text {Sil }}$ & $a_{\text {Crys }}$ & Sil/Crys & $T_{\text {Sil }}$ & $T_{\text {Crys }}$ & $\tau_{\text {ext }}$ \\
\hline Model A & 6800 & 250 & 320 & 5000 & 500 & 700 & 0.80 & 205 & 236 & 3.6 \\
Model B & 6800 & 150 & 195 & 5000 & 500 & 700 & 0.80 & 377 & 458 & 3.6 \\
Model C & 6800 & 50 & 67 & 5000 & 500 & 700 & 0.80 & 377 & 458 & 3.6 \\
Model D & 2500 & 5 & 5 & 1000 & 1000 & 1000 & 0.10 & 1101 & 1095 & 5.6 \\
Model E & 6800 & 250 & 320 & 5000 & 500 & 700 & 0.80 & 220 & 240 & 3.0 \\
Model F & 6500 & 551 & 300 & 10000 & 1000 & 1000 & 0.90 & 148 & 161 & 1.5 \\
Model G & 6500 & 477 & 300 & 10000 & 1000 & 1000 & 0.90 & 172 & 165 & 1.5 \\
Model H & 6500 & 390 & 300 & 10000 & 1000 & 1000 & 0.90 & 205 & 176 & 1.5 \\
\hline \hline & $T_{\text {eff }}$ & $R_{\text {Sil }_{1}}$ & $R_{\mathrm{Sil}_{2}}$ & $R_{\mathrm{o}}$ & $a_{\mathrm{Sil}_{1}}$ & $a_{\mathrm{Sil}_{2}}$ & $\mathrm{Sil}_{1} / \mathrm{Sil}_{2}$ & $T_{\mathrm{Sil}_{1}}$ & $T_{\mathrm{Sil}_{2}}$ & $\tau_{\mathrm{ext}}$ \\
\hline Model I & 6500 & 300 & 340 & 10000 & 1000 & 920 & 0.15 & 146 & 167 & 1.4 \\
\hline
\end{tabular}

about $70 \%$ of the total energy. Beyond $\lambda=7 \mu \mathrm{m}$ the total energy is almost all due to the thermal radiation by dust grains, mainly crystalline grains, which contribute about $70-80 \%$ at all wavelengths $(\lambda \lambda 7-100 \mu \mathrm{m})$. Model B (long-dashed line) has $R_{\mathrm{Sil}}=150 R_{*}$ and $R_{\mathrm{Crys}}=195 R_{*}$ and the temperature of the hottest grains are $T_{\mathrm{Sil}}=377 \mathrm{~K}$ and $T_{\mathrm{Crys}}=458 \mathrm{~K}$. In this model, the contribution of dust grains to the total energy begins at $\lambda=3.4 \mu \mathrm{m}$ (diffuse energy $\approx 55 \%$ and thermal energy $\approx 45 \%$ ). Above $\lambda=5 \mu \mathrm{m}$ thermal energy emitted by dust grains is responsible for the total energy observed. Finally model C (dashed line) was calculated with $R_{\mathrm{Sil}}=50 R_{*}$ and $R_{\text {Crys }}=67 R_{*}$ and, as a result, the temperature distribution for silicate grains vary between $T_{\mathrm{Sil}}=87 \mathrm{~K}-1022 \mathrm{~K}$ and $T_{\text {Crys }}=76-976 \mathrm{~K}$. The contribution of thermal energy by dust grains is already important at $\lambda=2.2 \mu \mathrm{m}$ (thermal energy $\approx 70 \%$ and diffuse energy $\approx 25 \%$ ). However, the overall fit is not good, and this result stresses that hot grains are needed to describe the SED at NIR wavelengths.

Figure 12b presents two completely different models: Model D represents a cool oxygen-rich AGB star with a thick dust envelope and model E represent a post-AGB star with a thin $\left(\tau_{1 \mu \mathrm{m}}=1.05\right)$ and a very detached dust envelope $\left(R_{\mathrm{Sil}}=250 R_{*}\right)$. Model D cannot describe the observations of IRAS $19386+0155$ and model E fits only the shortest $(\lambda<$ $1.65 \mu \mathrm{m})$ and longest wavelengths $(\lambda>10 \mu \mathrm{m})$. Again the NIR could not be fitted.

Figure 13 presents three models in which changed only the distances of the silicate grains to the central stars (inner radii, $\left.R_{\mathrm{Sil}}\right)$. In addition to these models this figure shows a model with amorphous silicate grains only (model I). In all models crystalline grains are closer to the central stars than silicate grains, and other parameters were kept (see Table 3). Similar to model E, these models can describe only the shortest and longest wavelengths (Fig. 13a). In contrast, model I fits only the longest wavelengths. In Fig. 13b we show an enlargement of the $\lambda \lambda 10-45 \mu \mathrm{m}$ region. Models $\mathrm{G}$ and $\mathrm{I}$ can give a better description of this region than other models, which were fitted with cool dust grains.

\subsection{Discussion: The infrared analysis of IRAS $19386+0155$}

The main spectral features were shown in the previous section. They reveal that IRAS $19386+0155$ has an oxygen-rich chemistry. The observation of molecular absorption lines of $\mathrm{H}_{2} \mathrm{O}$ (Fig. 10b) indicates the presence of a rather warm molecular envelope close to the photosphere. Similar features are observed in envelopes of oxygen-rich AGB stars (e.g., Tsuji et al. 1997). Another characteristic of mass-losing oxygen-rich AGB stars is the maser emission, the most important are $\mathrm{SiO}$, $\mathrm{H}_{2} \mathrm{O}$ and $\mathrm{OH}$. The $\mathrm{SiO}$ and $\mathrm{H}_{2} \mathrm{O}$ masers disappear at the end of AGB evolution, when the large amplitude pulsations and heavy mass loss cease. $\mathrm{OH}$ masers persist longer and some PNe still have $\mathrm{OH}$ maser emission. Lewis (2000) detected $\mathrm{OH}$ maser emission in IRAS $19386+0155$ but the $\mathrm{SiO}$ maser was not detected at all, which suggests that this star has already left the AGB. Further evidence of the post-AGB nature of this object was found to has $\Delta V=52 \mathrm{~km} \mathrm{~s}^{-1}$ from the $\mathrm{OH}$ maser observations which leads to an expansion velocity of $26 \mathrm{~km} \mathrm{~s}^{-1}$, higher than in an average AGB wind. This suggests that there is a fast wind emanating from the central star.

Concerning the dust features, the amorphous silicate band at $9.7 \mu \mathrm{m}$ seems to be absent (or in self-absorption) in ISO SWS spectra and there is an excess of emission at about $20 \mu \mathrm{m}$. When mass loss stops, the inner hot part of the shell will not be replenished by new hot dust, and moves outward and cools to temperatures of $150-200 \mathrm{~K}$ in less than $100 \mathrm{yr}$. At the same time the circumstellar shell becomes optically thin and the central star starts to shine through. According to Bedijn (1987) this will already happen 50-150 yr after the end of the AGB phase. His models shown that the $9.7 \mu \mathrm{m}$ feature completely disappears 200-600 yr after the end of the AGB phase. 

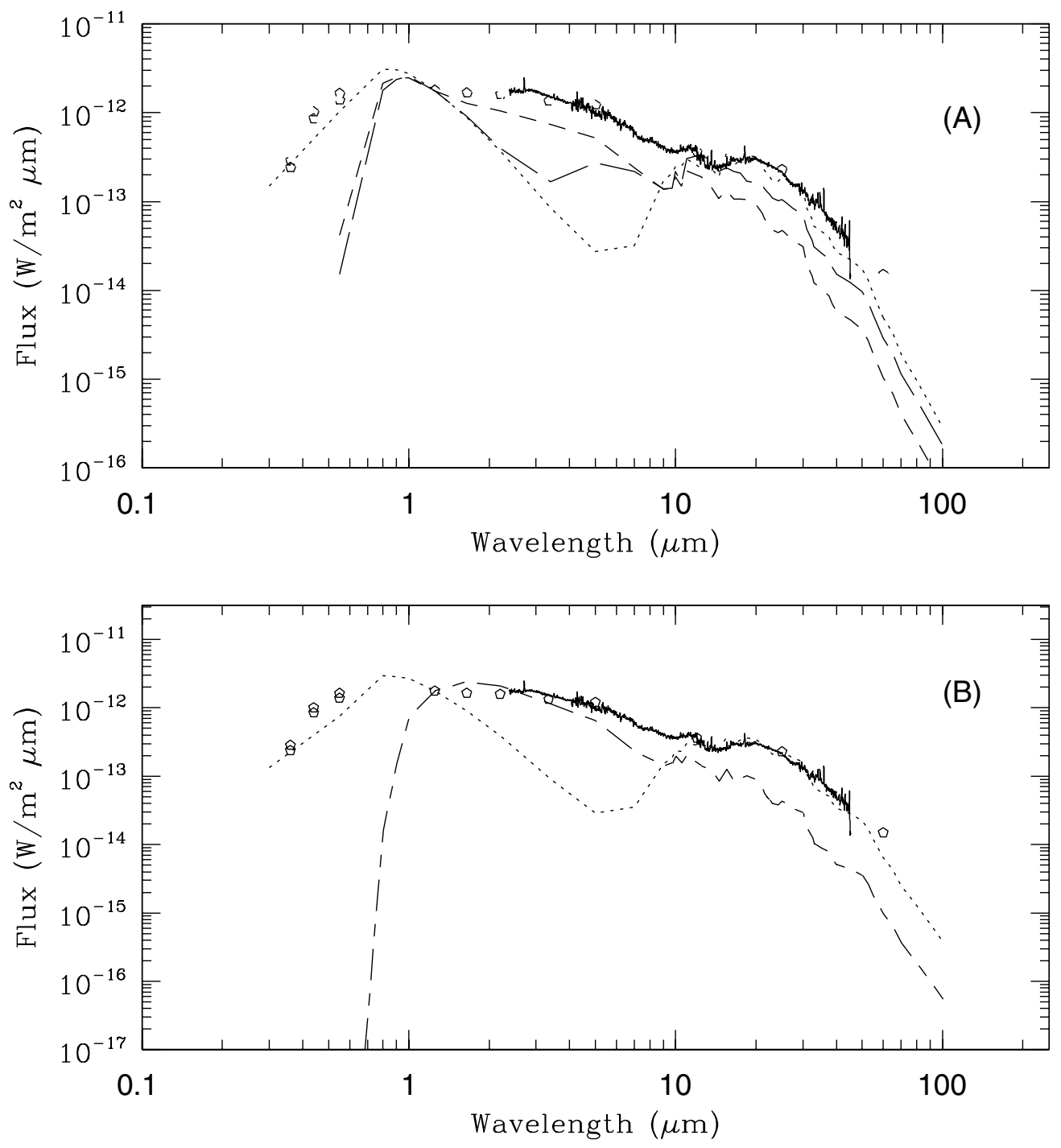

Fig. 12. a) Variation of the inner radii of the dust envelope. Model A (dotted line): $R_{\text {sil }}=250 R_{*}$ and $R_{\text {Cris }}=320 R_{*} ;$ model B (long-dashed line): $R_{\text {sil }}=150 R_{*}$ and $R_{\text {Cris }}=195 R_{*}$; model C (dashed line): $R_{\text {sil }}=50 R_{*}$ and $R_{\text {Cris }}=67 R_{*}$. b) Model D (long-dashed short-dashed lines) represents an oxygen-rich AGB star. Model E (dotted line) represent a very detached dust envelope for a star with $T_{\text {eff }}=6800 \mathrm{~K}$.

ISO SWS spectra also show some emission features which are identified as coming from crystalline silicates grains. Crystalline silicates are found in the outflows of evolved stars and around young stellar objects. A complete analysis of crystalline silicates was carried out by Molster et al. (2002, and references therein). They studied a sample of stars in several evolutionary stages and classified as disk sources or outflow sources. They suggested that there is a distinction between the two classes: disk sources present narrower features. However the sharpness of the crystalline silicate features shows a large variation and may be related to properties of the crystalline silicates such as the presence of impurities, the shape of the dust grains and irregularities in the lattice structure. Concerning IRAS $19386+0155$, we cannot exclude the possibility that the crystalline silicates grains are located in a disk.

Contrary to other post-AGB stars, the SED of IRAS $19386+0155$ does not present a double peak distribution, it is broad. The near-infrared excess present at $4-5 \mu \mathrm{m}$ is produced by hot dust grains $\left(T_{\mathrm{d}} \approx 1000 \mathrm{~K}\right)$ probably located in a disk, as we could not reproduce the SED of IRAS $19386+0155$ with spherically symmetric models. Very recently Ueta \& Meixner (2003) developed a 2-D code to solve the radiative transfer in axisymmetric shells. A model of any parameters can gives a different NIR flux when viewed at different angles, i.e., an edge-on disk results in higher NIR flux (hotter grains) than a face-on disk. However, at longer wavelengths the energy excess can be produced by cooler grains $\left(T_{\mathrm{d}} \approx 200 \mathrm{~K}\right.$, see model $\left.\mathrm{G}\right)$ which must be present in an approximately spherically symmetric outflow. Our modelling shows that the broad emission at $20 \mu \mathrm{m}$ can be described with cool silicate grains. The modelling also shows that the temperature obtained in the spectroscopic analysis (Sect. 3.2) can be used to represent the central star.

The presence of a disk is frequently associated with binarity phenomenon. If IRAS $19386+0155$ is a binary system, its component should be very cold, since no signs of its presence were detected in the optical spectrum. This would not be the first time that an unseen companion could be present near an 

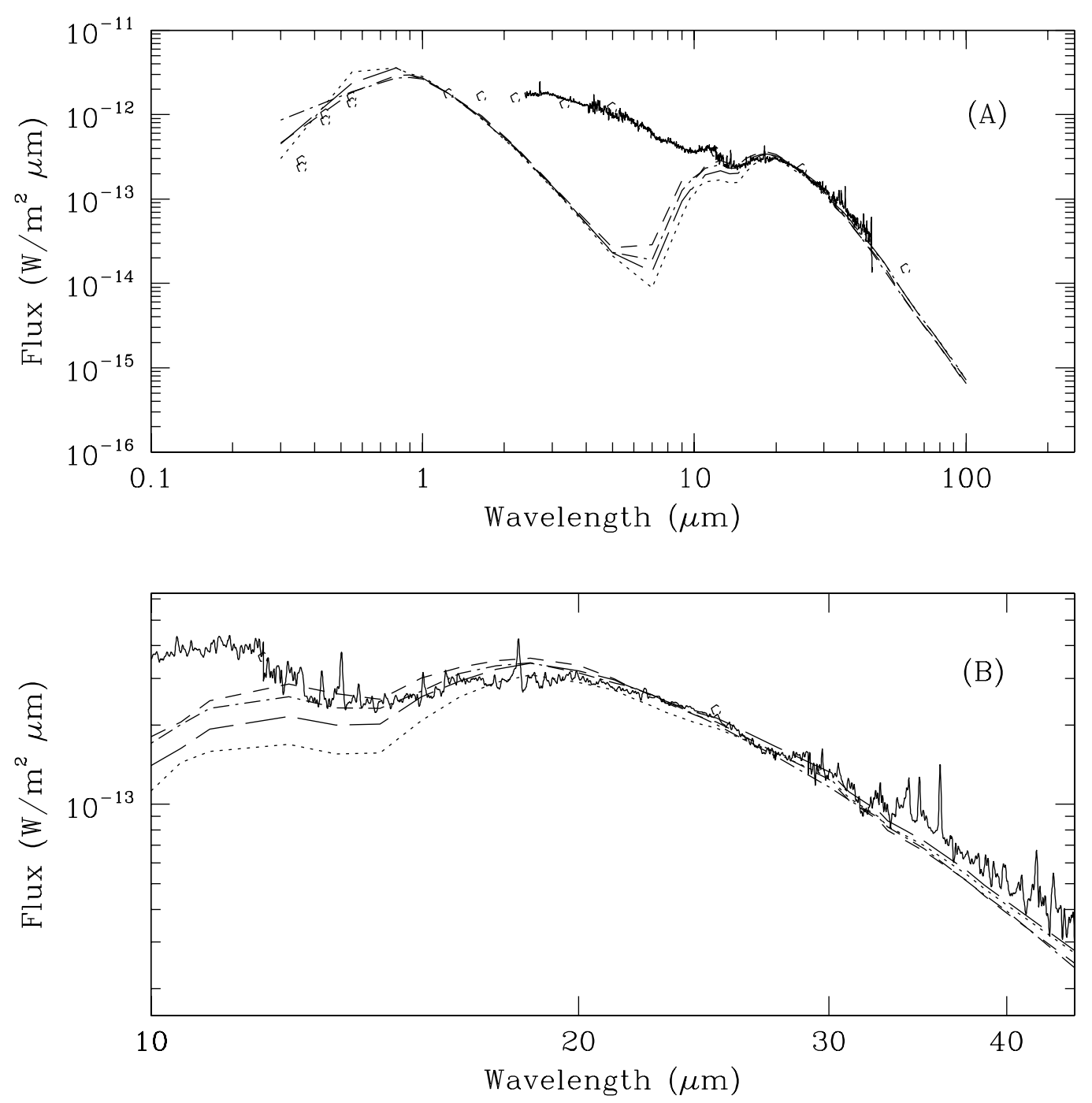

Fig. 13. a) Variation of $R_{\mathrm{Sil}}$ for a detached dust envelope with a central star with $T_{\text {eff }}=6500 \mathrm{~K}$. Model $\mathrm{F}$ (dotted line), $R_{\text {sil }}=551 R_{*}$; model G (long-dashed line), $R_{\text {sil }}=477 R_{*}$; model H (short-dashed line), $R_{\text {sil }}=390 R_{*}$. The inner radius for crystalline dust grains is fixed to $R_{\text {Crys }}=300 R_{*}$, closer to the central star than for silicate dust grains. Model I, with only amorphous silicate grains (dash-dotted line). b) An enlarged view of the 10-45 $\mu \mathrm{m}$ region. Models are the same as in Fig. 13a. These models can fit the continuum at about $20 \mu \mathrm{m}$.

F-type post-AGB star. In one of the observations of HD 172481 (Reyners \& van Winckel 2000) the presence of an M-type star was not revealed. The same occurred in AFGL 4106 (Molster et al. 1999). Its optical spectrum does not show any evidence of a cooler component. The binarity was revelead when the authors analysed the infrared ISO spectra where the presence of the cool component was confirmed due to the detection of several strong rotational-vibrational transitions of the $\mathrm{CO}$ and $\mathrm{OH}$ molecule. We have tried to find some trace of a binary nature of IRAS $19386+0155$ analysing the $2.4-5 \mu \mathrm{m}$ region of the ISO SWS spectra. As we know, in a circumstellar AGB envelope this region is dominated by strong absorption from several band heads of ro-vibrational molecular bands, such as $\mathrm{CO}$ first overtone and fundamental, $\mathrm{SiO}$ first overtone and $\mathrm{H}_{2} \mathrm{O}$ first overtone. Although we have found some of these bands, their strength is not enough to characterize an AGB star. They probably represent a fossil molecular envelope from the progenitor AGB star. So, using ISO SWS we do not see the presence of a companion. We suggest that the crystalline silicates observed in IRAS $19386+0155$ spectra are in a disk and the amorphous silicates are distributed in a spherically symmetric envelope.

\section{Conclusions}

Our optical and infrared spectroscopic analysis for the post-AGB star candidate IRAS $19385+0155$, shows that it is an oxygen-rich star displaying a similar abundance pattern to that of other stars already analysed, SAO 239832, HD 133656 and HR 4912, as can be seen in Figs. 6 and 7. The infrared analysis showed that the SED of IRAS $19386+0155$ does not show the double-peak emission seen in two post-AGB stars, HD 302821 (=IRAS 10215-5916 (Fig. 9b, this work) and in AFGL4106 (Molster et al. 1999).

The ISO SWS was used to characterize the chemical constitution present in the envelope of IRAS $19386+0155$. The star has an oxygen-rich envelope. From an analysis of the $2.4-5 \mu \mathrm{m}$ region in these spectra we have rejected the presence of a cold 
companion. Using ISO SWS spectra and NIR photometry we have shown that there exist both hot dust and cold dust in the circumstellar environment of this star. Our spherically symmetric models cannot reproduce the SED of IRAS 19386+0155 which is broad, and extensive suggesting the presence of a dust disk, which is probably seen edge-on.

Acknowledgements. S. Lorenz Martins acknowledges CNPq (478309/01-5) for financial support and the hospitality of the Observatorio Nacional/MCT where part of this research was carried out. This research was performed using the SIMBAD database operated at CDS, Strasbourg, France. We also thank the referee, Dr. K. Justtanont, for her critical reading of this paper and valuable advice.

\section{References}

Arkhipova, V. P., Ikonnikova, N. P., Noskova, R. I., \& Sokol, G. V. 2000, AstL, 26, 609

Bedijn, P. J. 1987, A\&A, 186, 136

Biémont, E., \& Godefroid, M. 1980, Phys. Scr., 22, 231

Bond, H., \& Luck, R. E. 1987, ApJ, 312, 203

Blöcker, T., \& Schönberner, D. 1990, A\&A, 240, L11

Cami, J. 2002, Ph.D. Thesis, Univ. of Amsterdam

David, P., \& Pégourié, B. 1995, A\&A, 293, 833

de Graauw, T., Haser, L. N., Beintema, D. A., et al. 1996, A\&A, 315, L49

Fouque, P., Le Bertre, T., Epchtein, N., Guglielmo, F., \& Kerschbaum, F. 1992, A\&AS, 93, 151

Gonzalez, G., \& Wallerstein, G. 1992, MNRAS, 254, 343

Gratton, R., \& Sneden, C. 1987, A\&A, 178, 179

Hannaford, P., Lowe, R. M., Grevesse, N., Biemont, E., \& Whaling, W. 1982, ApJ, 261, 736

Jäger, C., Molster, F. J., Dorschner, J., et al. 1998, A\&A, 339, 904

Kaufer, A., Stahl, O., Tubbesing, S., et al. 1999, The Mesenger, 95, 8

Kingsburgh, R. L., \& Barlow, M. J. 1994, MNRAS, 271, 257

Koike, C., Tsuchiyama, A., Shibai, H., et al. 2000, A\&A, 363, 1115

Kurucz, R. L. 1993, CD-ROM 13, Atlas9 Stellar Atmosphere Programs and $2 \mathrm{~km} \mathrm{~s}^{-1}$ Grid (Cambridge: Smithosian Astrophys. Obs.)

Lambert, D. L., Roby, S. W., \& Bell, R. A. 1982, ApJ, 254, 664
Lambert, D. L., Heath, J. E., Lemke, M., \& Drake, J. 1996, ApJS, 103, 183

Lewis, B. M. 2000, ApJ, 533, 959

Lorenz-Martins, S., \& Pompeia, L. 2000, MNRAS, 315, 856

Luck, R. E., \& Bond, H. 1985, ApJ, 292, 559

Luck, R. E., \& Lambert, D. 1985, ApJ, 298, 782

Luck, R. E., \& Bond, H. 1989, ApJS, 71, 559

Luck, R. E., Bond, H., \& Lambert, D. L. 1990, 357, 188

Luck, R. E. 1993, The chemical composition of luminous high-latitude stars post-AGB stars, ed. D. D. Sasselov, in Luminous HighLatitude Stars, 87

Martin, G. A., Fuhr, J. R., \& Wiese, W. L. 1988, J. Phys. Chem. Ref. Data, 17,4

McCausland, R. J. H., Colon, E. S., Dufton, P. L., \& Keenan, F. P. 1992, ApJ, 394, 298

Molster, F. J., Waters, L. B. F. M., Trams, N. R., et al. 1999, A\&A, 350,163

Molster, F. J., Waters, L. B. F. M., \& Tielens, A. G. G. M. 2002, A\&A, 382,222

Pereira, C. B., \& Junqueira, S. 2003, A\&A, 402, 1061

Reyniers, M., \& van Winckel, H., 2001, A\&A, 365, 465

Ryans, R. S. I., Dufton, P. L., Mooney, C. J., et al. 2003, A\&A, 401, 1119

Schönberner, D. 1983, A\&A, ApJ, 272, 708

Sloan, G. C., Kraemer, K. E., Price, S. D., \& Shipman, R. F. 2003, ApJSS, 147, 379

Sneden, C. 1973, Ph.D. Thesis, Univ. of Texas

Thévenin, F., Parthasarathy, M., Jasniewicz, G., et al. 2000, A\&A, 359,138

Trams, N. R., Waelkens, C., \& Waters, L. B. F. M. 1993, Extremely metal-poor post-AGB stars, ed. D. D. Sasselov, in Luminous High-Latitude Stars, 103

Tsuji, T., Ohnaka, K., Aoki, W., \& Yamamura, I. 1997, A\&A, 320, L1

Ueta, T., \& Meixner, M. 2003, ApJ, 586, 1338

van Winckel, H. 1997, A\&A, 319, 561

van Winckel, H., \& Reyniers, M. 2000, A\&A, 354, 135

van Winckel, H. 2003, ARA\&A, 41, 391

Venn, K. A. 1993, ApJ, 414, 316

Waelkens, C., Van Winckel, H., Bogaert, E., \& Trams, N. R. 1991, A\&A, 251, 495

Wiese, W. L., Smith, M. W., \& Miles, B. M. 1969, NBS Ref. Data Ser, 22,2 